\title{
Extinction of Disease Pathogenesis in Infected Population and Its Subsequent Recovery: A Stochastic Approach
}

\author{
Priti Kumar Roy, ${ }^{1}$ Jayanta Mondal, ${ }^{2}$ Rupa Bhattacharyya, ${ }^{3}$ \\ Sabyasachi Bhattacharya, ${ }^{4}$ and Tamas Szabados ${ }^{5}$ \\ ${ }^{1}$ Centre for Mathematical Biology and Ecology, Department of Mathematics, Jadavpur University, Kolkata, West Bengal 700032, India \\ ${ }^{2}$ Department of Mathematics, Barasat College, Kolkata 700126, India \\ ${ }^{3}$ Department of Chemistry, Narula Institute of Technology, Kolkata 700109, India \\ ${ }^{4}$ Agricultural and Ecological Research Unit, Indian Statistical Institute, 203 B. T. Road, Kolkata 700108, India \\ ${ }^{5}$ Department of Stochastics, Institute of Mathematics, Budapest University of Technology and Economics, Budapest 1521, Hungary
}

Correspondence should be addressed to Priti Kumar Roy; pritiju@gmail.com

Received 5 February 2013; Accepted 21 May 2013

Academic Editor: Xinyu Song

Copyright (C) 2013 Priti Kumar Roy et al. This is an open access article distributed under the Creative Commons Attribution License, which permits unrestricted use, distribution, and reproduction in any medium, provided the original work is properly cited.

\begin{abstract}
A stochastic mathematical model of host-pathogen interaction has been developed to estimate the time to extinction of infected population. It has been assumed in the model that the infected host does not grow or reproduce but can recover from pathogenic infection and move to add to the susceptible host population using various drugs or vaccination. Extinction of infected population in host-pathogen interaction depends significantly upon the total population. Here, we consider an extension of our previous work with the stochastic approach to predict the time to extinction of disease pathogenesis. The optimal control approach helped in designing an innovative, safe therapeutic regimen where the susceptible host population enhanced with simultaneous decrease in the infected population. By means of an optimal control theory paradigm, it has also been shown in our preceding research paper that the cost-effective combination of treatment may depend on the population size. In this research paper, we have studied an approximation which is derived in favor of quasi-stationary distribution along with the expected time to extinction for the model of host-pathogen interactions. The complete study has been roofed through the stochastic approach in context that disease pathogenesis is to be extinct and infected population are going to be recovered. Numerical simulation is also done to confirm the analysis.
\end{abstract}

\section{Introduction}

The modeling of epidemic diseases is an age-old problem [1] and makes a sincere effort in understanding the development of mathematical models for epidemics from the 18th century to the present day. These models are shown to be of used in predicting and controlling the spread of infection. But in the recent context of epidemiological research, microbial pathogenesis reflects the interaction between two entities, host and pathogen, which is somewhat related to the predatorprey model $[2,3]$. These classes of models are also relevant for host-parasite type of models. Host-pathogen models are mathematical prototypes pertaining to epidemiology, and are of immense importance in view of the emergence and reemergence of epidemiological diseases in the present day global scenario $[4,5]$.

Application of mathematical concepts and techniques to analyze host-pathogen interactions was done by various researchers [6-8]. A very recent study throws light on the antiviral drug treatment along with immune activator IL-2 and optimal control in disease pathogenesis by deterministic model formulation [9]. In this research paper, a conventional host-pathogen model has been considered including the recovery of the infected individuals to the healthy susceptible organisms. In this case, the host population is divided into two classes: susceptible $(S)$, that is, healthy organisms, and infected individuals $(I)$. Pathogens $(V)$ cause infection to host population by transforming $S$ to $I$. Over the last 
several years, many researchers focused their attention on the mathematical and biological aspects of host-pathogen interactions. Beltrami and Carroll [10] as well as Venturino, worked on the role of viral disease considering a three-species model of susceptible and infected phytoplankton as well as their predator [4]. Hethcote and Driessche [11] formulated SIS epidemiological models where delay has been incorporated corresponding to the infectious period and disease-related deaths. Another pioneering work was reported by [12] where the transport of coevolved host-pathogen systems into new environment leads to the evolution of altered levels of pathogen aggressiveness, if transmission rates are different in the new environment. With the growing research in the preypredator and other prototypical systems, it is apparent that the pathogen or viral growth through replication influences the model dynamics. This has been emphasized by Bairagi et al. in a subsequent communication [13]. In another pioneering communication in recent years, Bairagi et al. carried a comparative study of the prey-predator model with several response functions [14].

Epidemiological modeling uses both deterministic and stochastic approaches in host-pathogen interactions [15]. Both model types have their respective advantages and weaknesses. Deterministic models may be considered as an approximation of a corresponding stochastic model. An important difference between the two is that the stochastic model deals with a finite population size, while the deterministic model deals only with proportion [16]. The deterministic version of the model can be derived as an approximation of the stochastic version using the state variables which are susceptible host $(S)$, infected host $(I)$, and pathogen $(V)$ where the total population comprising the susceptible and infected host is $N$. Recurrence can be explained by the combined influence of epidemic and demographic forces. In stochastic models, infection will eventually become extinct, and time to extinction is an important measure of the persistence of the infection [17].

This paper specifies a stochastic approach of our earlier work [18] designed for some widespread infection in closed population. It is a well-known fact that beyond a threshold value, that is, basic reproduction ratio, which is determined by the parameters of the model, the deterministic model predicts that the proportion of infected individuals will approach a positive endemic level as time approaches infinity. However, the stochastic model predicts that the infection will become extinct. Generally, stochastic version of the model is compared with prey-predator interaction or more precisely the host-pathogen interaction, and thus the concept of the quasi-stationary state is an important aspect of this model [19]. It should be mentioned here that the stochastic logistic model can be interpreted as SIS model and that SIS model is used for infection that gives no immunity. In this case, it is assumed that an individual who recovers from an infection remains susceptible to further infection. The time to extinction from quasi-stationary distribution is a measure of the persistence of the infection. The stationary distribution is to be an additional importance since the expected time to extinction from quasi-stationary can be expressed in terms of this distribution. A major goal of the analysis of the stochastic model is therefore to derive an approximation of the quasistationary distribution. This derivation is based on a diffusion approximation of the stochastic discrete state model.

The model is analyzed in two different avenues, analytical and numerical. In our research paper, we have found an approximation for the marginal distribution of the infected population in quasi-stationary condition and its time to extinction. The time to extinction of the infected population depends on the total population, and the study has been carried out through stochastic approach so that infected population is to be extinct and increase the susceptible population. However, being an extension of our previous work [18], where deterministic model was used and optimal control theory applied to predict the decrease of infected population along with cost-effective combination of treatment, our present work emphasizes on the time to extinction of the infected host population or the disease by stochastic model formulation and its subsequent analysis and evaluation.

\section{The Deterministic Model}

We consider the three components of the basic threedimensional deterministic host pathogen model [18] consisting of a host population, whose concentration is denoted by $N([N]=$ number of host per designated area $)$ and a pathogen population inflicting infection in the host population whose concentration is denoted by $V([V]=$ number of pathogens per designated area). The following differential equations are formed at initial dynamics on the change of host pathogen interaction with time $t$ :

$$
\begin{gathered}
\frac{d S}{d t}=r S\left(1-\frac{S+I}{K}\right)-\lambda S I-\frac{\gamma S V}{h_{\gamma}+S}+\delta I, \\
\frac{d I}{d t}=\lambda S I+\frac{\gamma S V}{h_{\gamma}+S}-d_{I} I-\delta I, \\
\frac{d V}{d t}=-\frac{\gamma S V}{h_{\gamma}+S}+\eta d_{I} I-\mu V .
\end{gathered}
$$

Here in the presence of pathogenic infection, the host population is divided into two disjoint classes, susceptible host $S$ and infected host $I$. In the ideal case of no pathogen, the growth of susceptible host population follows the logistic law [19] implying that this growth is entirely controlled by an intrinsic birth rate constant $r\left(\in R_{+}\right)$with a carrying capacity $K\left(\in R_{+}\right) \cdot \gamma\left(\in R_{+}\right)$is the force of infection through contact with pathogens, and pathogens maximally infect $\gamma$ susceptible hosts per day. This infection rate is half maximal at susceptible host population density of $h_{\gamma}$ host. $\lambda\left(\in R_{+}\right)$is the intensity of infection by infected host, and $d_{I}\left(\in R_{+}\right)$is the death rate constant of $I$. Rate of cell lysis (replication of pathogens) is $\eta\left(\in R_{+}\right)$, and the natural death rate of pathogens is denoted as $\mu\left(\in R_{+}\right)$. We assume that the infected hosts do not grow or reproduce, but they can recover from pathogenic infection and move to the susceptible host population. Such recovery would stem out from immunization or vaccination. We consider a recovery rate of infected host $(I)$ to be denoted by $\delta\left(\in R_{+}\right)$. 
In spirit of Bonhoefer et al., we employed a simplified system with two components, the susceptible and the infected host. It is assumed that at equilibrium point $\dot{V}=0$, so $V$ can be eliminated by putting $V=\eta d_{I}\left(h_{\gamma}+S\right) I /\left(\mu\left(h_{\gamma}+S\right)+\gamma S\right)$. With this choice of $V$ model, (1) reduces to

$$
\begin{gathered}
\frac{d S}{d t}=r S\left(1-\frac{S+I}{K}\right)-\lambda S I-\alpha \frac{S I}{\beta+S}+\delta I, \\
\frac{d I}{d t}=\lambda S I+\alpha \frac{S I}{\beta+S}-d_{I} I-\delta I,
\end{gathered}
$$

where $\gamma \eta d_{I} /(\gamma+\mu)=\alpha$ and $h_{\gamma} \mu /(\gamma+\mu)=\beta$.

An alternative deterministic formulation of the reduced model (2), one assumes birth and death rate functions $B(S)$ and $D(S)$, respectively, of

$$
B(S)=b_{1} S-b_{2}(S+I) S, \quad D(S)=d_{1} S+d_{2}(S+I) S,
$$

where $b_{1}, b_{2}$ and $d_{1}, d_{2}>0$. Here, $b_{1}$ and $d_{1}$ are intrinsic rate, $b_{2}$ and $d_{2}$ are crowding coefficient [20], and thus intrinsic growth rate $(r)$ and carrying capacity $(K)$ are defined as follows: $r=b_{1}-d_{1}$ and $K=\left(b_{1}-d_{1}\right) /\left(b_{2}+d_{2}\right)$ [20],

$$
\begin{gathered}
\frac{d S}{d t}=(B(S)-D(S))-\lambda S I-\alpha \frac{S I}{\beta+S}+\delta I, \\
\frac{d I}{d t}=\lambda S I+\alpha \frac{S I}{\beta+S}-\left(d_{I}+\delta\right) I .
\end{gathered}
$$

\section{The Stochastic Model Formulation}

There are two state variables, namely, the number of susceptible hosts $S(t)$ and the number of infected hosts $I(t)$ at time $t$. They jointly take values in the state space $S_{p}=\{(m, n): m=$ $0,1,2, \ldots ; n=0,1,2, \ldots\}$. The joint distribution of $S(t)$ and $I(t)$ at time $t$ is denoted by

$$
p_{m n}(t)=P\{S(t)=m, I(t)=n\} .
$$

We use this notation even when $m$ and/or $n$ are negative, with the convention that $p_{m n}(t)$ is equal to zero. The model is based on the following four basic events, that is, birth of a susceptible host, death of a susceptible host, infection of an uninfected host, and death or recovery of an infected host. The transition rates of the model are shown in Table 1.

\section{Description of the Transition States}

The total number of population $N$ is increased by unity, if there is a birth of susceptible host $(S)$ for a small time interval $\Delta t$. But to make the population to be the same, we should assume that there must be a natural death of susceptible host $(S)$. These phenomena are captured through the first two rows of the transition matrix. On the other hand, if there is an infection in the susceptible host it can be balanced by an increase of an infected host, $(I)$. The susceptible class is infected either by direct infection with the pathogen or by replication of viral generated within the infected population.
This occurs one at a time, and so the increases of the infected class are reflected by the rise of unity in the transition state. If there is an infected host, the natural death should be reflected through a natural birth of susceptible host. At the end, naturally, the recovery of infected host must be reinstated to the susceptible host.

\section{Formulation of Kolmogorov's Forward Equation}

We are supposing that in a time interval of infinitesimally little length $(\Delta t)$, the probability of precisely one birth (or one death) is birth rate (or death rate) $\times(\Delta t)+$ intuitively more than one occasion (birth and/or death) in $o(\Delta t)$. We as well believe the prospect $p_{m n}(t+\Delta t)$, where $\Delta t \downarrow 0$. The Kolmogorov's forward equations for the representation can be written as

$$
\begin{aligned}
p_{m, n}(t+\Delta t)= & \lambda_{1}(m-1, n) p_{m-1, n}(t) \Delta t \\
& +\mu_{1}(m+1, n) p_{m+1, n}(t) \Delta t \\
& +\mu_{2}(m, n+1) p_{m, n+1}(t) \Delta t \\
& +\gamma_{2}(m+1, n-1) p_{m+1, n-1}(t) \Delta t \\
& +(1-\kappa(m, n) \Delta t) p_{m n}(t)+o(\Delta t),
\end{aligned}
$$

where

$$
\kappa(m, n)=\lambda_{1}(m, n)+\mu_{1}(m, n)+\mu_{2}(m, n)+\gamma_{2}(m, n) .
$$

Note that all proceedings consisting of more than one birth or more than one death are incorporated in the $o(\Delta t)$ expression

$$
\begin{aligned}
\therefore p_{m, n}^{\prime}(t)= & \lim _{\Delta t \rightarrow 0} \frac{p_{m, n}(t+\Delta t)-p_{m, n}(t)}{\Delta t} \\
= & \lambda_{1}(m-1, n) p_{m-1, n}(t) \\
& +\mu_{1}(m+1, n) p_{m+1, n}(t) \\
& +\mu_{2}(m, n+1) p_{m, n+1}(t) \\
& +\gamma_{2}(m+1, n-1) p_{m+1, n-1}(t) \\
& -\kappa(m, n) p_{m n}(t) .
\end{aligned}
$$

\section{The Quasi-Stationary Distribution}

We obtain primarily a deferential equation that will be used afterward in this subsection and in the discussion of time to extinction in the subsequent subsection. Place $n=0$ in Kolmogorov's Forward equations (8), and put in the explicit expressions for the evolution rates given above. In addition, commence

$$
p_{.1}(t)=\sum_{m=0}^{\infty} p_{m n}=P\{I(t)=n\}
$$


TABLE 1: Hypothesized transition rates for the stochastic version.

\begin{tabular}{lcc}
\hline Event & Transition & Transition rates \\
\hline Birth of a susceptible host & $(m, n) \rightarrow(m+1, n)$ & $\lambda_{1}(m, n)=b_{1} m-b_{2}(m+n) m+\delta n$ \\
Death of a susceptible host & $(m, n) \rightarrow(m-1, n)$ & $\mu_{1}(m, n)=d_{1} m+d_{2}(m+n) m$ \\
Infection of an uninfected host & $(m, n) \rightarrow(m-1, n+1)$ & $\gamma_{2}(m, n)=(\lambda+(\alpha /(\beta+m))) m n$ \\
Death or recovery of an infected host & $(m, n) \rightarrow(m, n-1)$ & $\mu_{2}(m, n)=\left(d_{I}+\delta\right) n$ \\
\hline
\end{tabular}

to indicate the subsidiary allocation of the number of infected individuals at time $t$. By summing the forward equations intended for $n=0$ over all $m$-values, we acquire

$$
p_{.0}^{\prime}(t)=\left(\delta+d_{I}\right) p_{.1}(t) .
$$

After that, we develop an organization of equations for the quasi-stationary distribution. The state possibility conditioned on not being engrossed is signified $q_{m n}(t)$ and specified by

$$
\begin{aligned}
q_{m n}(t) & =P\{S(t)=m, I(t)=n \mid I(t) \neq 0\} \\
& =\frac{p_{m n}(t)}{1-p_{.0}(t)}, \quad m=0,1,2, \ldots, n=1,2, \ldots
\end{aligned}
$$

We initiate $q_{. n}(t)=\sum_{m=0}^{\infty} p_{m n}(t)$ to stand for the marginal distribution for the number of infected individuals at time $t$, habituated on not having attained any condition in the engrossing position. By differentiating the expression for $q_{m n}(t)$ in (11) and relating (10), we get hold of

$$
q_{m n}^{\prime}(t)=\frac{p_{m n}^{\prime}(t)}{1-p_{.0}(t)}+\left(\delta+d_{I}\right) q_{.1}(t) \frac{p_{m n}(t)}{1-p_{.0}(t)}
$$

By pertaining the forward equations for $q_{m n}(t)$ in (8), we achieve the following scheme of differential equations for the conditional state probabilities $q_{m n}(t)$ :

$$
\begin{aligned}
\therefore q_{m n}^{\prime}(t)= & \lambda_{1}(m-1, n) q_{m-1, n}(t) \\
& +\mu_{1}(m+1, n) q_{m+1, n}(t) \\
& +\mu_{2}(m, n+1) q_{m, n+1}(t) \\
& +\gamma_{2}(m+1, n-1) q_{m+1, n-1}(t) \\
& -\kappa(m, n) q_{m n}(t)+\left(\delta+d_{I}\right) q_{.1}(t) q_{m n}(t), \\
& \quad m=0,1,2, \ldots, \quad n=1,2, \ldots
\end{aligned}
$$

The quasi-stationary distribution $q_{m n}(t)$ is the stationary solution of this system of equations.

\section{The Distribution of the Time to Extinction}

Two initial distributions are principally fascinating. One is the quasi-stationary distribution, and another communicates to one infected individual. The distribution of the time to extinction $\tau$ can be unwavering if we can resolve the Kolmogorov forward equations (8) for $p_{m n}(t)$. The reason is that the happening that $\tau$ surpasses $t$ is equal to the event that the number of infected individuals at time $t$ is positive. By allowing for the balancing events we attain

$$
P\{\tau \leq t\}=P\{I(t)=0\}=p_{.0}(t) .
$$

Therefore, the cumulative distribution function of the time to extinction at time $t$ equals the marginal possibility in which the number of infected individuals at time $t$ equals 0 . The distribution of the time to extinction is particularly straightforward when the opening distribution is equal to the quasi-stationary distribution. Let us indicate the time to extinction from quasi-stationarity by $\tau_{Q}$ explicitly. We demonstrate that $\tau_{Q}$ has an exponential distribution and that its predictable value is equal to

$$
E\left(\tau_{\mathrm{Q}}\right)=\frac{1}{\delta+d_{I}} q_{.1}(t)
$$

To gain this consequence, we put $q_{m n}^{\prime}(t)=0$ in (12). Thus, we are guided to the initial value problems

$$
\begin{array}{r}
p_{m n}^{\prime}=-\left(\delta+d_{I}\right) q_{.1} p_{m n}, \quad p_{m n}(0)=q_{m n} \\
m=0,1,2 \ldots, \quad n=1,2,3 \ldots
\end{array}
$$

with solutions

$$
\begin{aligned}
& p_{m n}=q_{m n} \exp \left(-\left(\delta+d_{I}\right) q_{.1} t\right), \\
& m=0,1,2 \ldots, \quad n=1,2,3 \ldots
\end{aligned}
$$

By adding these expressions of $p_{m n}(t)$ over all $m$, we obtain

$$
p_{. n}=q_{. n} \exp \left(-\left(\delta+d_{I}\right) q_{.1} t\right), \quad n=1,2,3 \ldots
$$

The differential equation for $p_{.0}(t)$ in (10) can now be answered since the right-hand side of this equation is known from above. Remembering that we have the initial value $p_{.0}(0)=0$, we acquire $p_{.0}(t)=1-\exp \left(-\left(\delta+d_{I}\right) q_{.1} t\right)$. This institutes the claim that $\tau_{Q}$ has an exponential distribution with expected value agreed by (15).

\section{Diffusion Approximation and the Approximation of Quasi-Stationary Distribution}

In this section, we have derived the diffusion approximation for the process formulated in Section 2. In order to approximate the quasi-stationary distribution, we consider 
TABLE 2: Possible changes in the two-population system (4) with the probabilities.

\begin{tabular}{lc}
\hline Change & Probability \\
\hline$\Delta x_{1}=[1,0]^{T}$ & $p_{1}=\left(\left(b_{1}-b_{2}(S+I)\right) S+\delta I\right) \Delta t$ \\
$\Delta x_{2}=[-1,0]^{T}$ & $p_{2}=\left(d_{1}+d_{2}(S+I)\right) S \Delta t$ \\
$\Delta x_{3}=[-1,1]^{T}$ & $p_{3}=(\lambda+\alpha /(\beta+S)) S I \Delta t$ \\
$\Delta x_{4}=[0,-1]^{T}$ & $p_{4}=\left(d_{I}+\delta\right) I \Delta t$ \\
\hline
\end{tabular}

the two-dimensional process which is represented by the set of differential equation (4)

$$
\begin{gathered}
\frac{d S}{d t}=(B(S)-D(S))-\lambda S I-\alpha \frac{S I}{\beta+S}+\delta I, \\
\frac{d I}{d t}=\lambda S I+\alpha \frac{S I}{\beta+S}-d_{I} I-\delta I .
\end{gathered}
$$

The main result is the quasi-stationary distribution is approximated by a bivariate normal distribution, if $N$ is sufficiently large.

The critical point of the rescaled deterministic model that corresponds to a pathogenic infection is denoted by $x=$ $(\widehat{S}, \widehat{I})$, where

$$
\widehat{S}=\frac{\left(d_{I}+\delta-\alpha-\lambda \beta\right)+\sqrt{\left(d_{I}+\delta-\alpha-\lambda \beta\right)^{2}+4 \beta \lambda\left(\delta+d_{I}\right)}}{2 \lambda},
$$

$$
\widehat{I}=\frac{r \widehat{S}(K-\widehat{S})}{r \widehat{S}+d_{I} K}
$$

The changes in the scaled state variables $S$ and $I$ during the time interval from $t$ to $t+\Delta t$ are denoted by $\Delta S$ and $\Delta I$ using Table 2: $\Delta S=S(t+\Delta t)-S(t)$ and $\Delta I=I(t+\Delta t)-I(t)$. From the hypotheses of the original process, we determine the mean and the covariance of the vector with components $\Delta S$ and $\Delta I$. We begin with the mean

$$
\begin{aligned}
E\left(\begin{array}{c}
\Delta S \\
\Delta I
\end{array}\right)= & \left(\begin{array}{c}
r S\left(1-\frac{S+I}{K}\right)-\lambda S I-\alpha \frac{S I}{\beta+S}+\delta I \\
\lambda S I+\alpha \frac{S I}{\beta+S}-d_{I} I-\delta I
\end{array}\right) \Delta t \\
& +o(\Delta t)=b(x) \Delta t+o(\Delta t) .
\end{aligned}
$$

The Jacobian matrix of the vector $b(x)$ with respect to $x$ is denoted by $B(x)$

$$
B(\widehat{x})=\frac{\delta b(\widehat{x})}{\delta x}=\left(\begin{array}{cc}
A_{1} & A_{2} \\
A_{3} & 0
\end{array}\right)
$$

where $A_{1}=r-(2 r \widehat{S} / K)-(r \widehat{I} / K)-\lambda \widehat{I}-\left(\alpha \beta \widehat{I} /(\widehat{S}+\beta)^{2}\right), A_{2}=$ $-(r \widehat{S} / K)-d_{I}$, and $A_{3}=\widehat{I}\left(\lambda+\left(\alpha \beta /(\beta+\widehat{S})^{2}\right)\right)$.
Next, we determine the covariance matrix of the vector of changes in the state variables during the time interval $(t, t+$ $\Delta t)$

$$
\begin{aligned}
\operatorname{Cov}\left(\begin{array}{c}
\Delta S \\
\Delta I
\end{array}\right)= & \left(\begin{array}{cc}
2\left[b_{1} S-b_{2}(S+I) S+\delta I\right] & -\left(\delta+d_{I}\right) I \\
-\left(\delta+d_{I}\right) I & 2\left(\delta+d_{I}\right) I
\end{array}\right) \Delta t \\
& +o(\Delta t) \\
= & M(x) \Delta t+o(\Delta t) .
\end{aligned}
$$

The matrix $M(x)$ is approximated by evaluating it at the critical point $(\widehat{S}, \widehat{I})$ corresponding to the pathogenic infection level

$$
M(\widehat{x})=\left(\begin{array}{cc}
A_{4} & A_{5} \\
A_{5} & -2 A_{5}
\end{array}\right)
$$

where $A_{4}=2\left[b_{1} \widehat{S}-b_{2}(\widehat{S}+\widehat{I}) \widehat{S}+\delta \widehat{I}\right]$ and $A_{5}=-\left(\delta+d_{I}\right) \widehat{I}$.

For large $N$, the process $N^{1 / 2}(x-\widehat{x})$ is approximated by a stable bivariate Ornstein-Uhlenbeck process, with local drift matrix $B(\widehat{x})$ and local covariance matrix $M(\widehat{x})$. Then, the stationary distribution of the Ornstein-Uhlenbeck process is bivariate normal with mean 0 and variance $\sum=\left(\begin{array}{ll}\sigma_{1} & \sigma_{2} \\ \sigma_{2} & \sigma_{3}\end{array}\right)$ through the relationship

$$
B(\widehat{x}) \sum+\sum B^{T}(\widehat{x})=-M(\widehat{x})
$$

where the superscript $T$ is used to denote the transpose. After solving the above equation, we get

$$
\begin{aligned}
& \sigma_{1}=\frac{A_{3} A_{4}+2 A_{2} A_{5}}{2 A_{1} A_{3}}, \\
& \sigma_{2}=\frac{2 A_{2} A_{5}+A_{4} A_{3}-2 A_{1} A_{3}-2 A_{1}^{2} A_{5}}{2 A_{1} A_{2} A_{3}}, \\
& \sigma_{3}=\frac{A_{5}}{A_{3}} .
\end{aligned}
$$

Note that the parameter should also satisfy $\sigma_{1}>0, \sigma_{3}>$ 0 . Thus, the diffusion approximation led to the conclusion that the marginal distribution of the infected population size in quasi-stationarity is approximately $q_{. n}$. To achieve consistency with the fact $I \geq 0$, the approximating normal distribution is modified by truncation at $1 / 2$. Hence, we have the following approximation

$$
q_{. n} \approx \frac{1}{\sqrt{\sigma_{3} / N}} \frac{\phi\left((n-\widehat{I}) / \sqrt{\sigma_{3} / N}\right)}{\Phi\left((\widehat{I}-0.5) / \sqrt{\sigma_{3} / N}\right)}
$$

where $\Phi$ and $\phi$ are, respectively, the standard normal c.d.f. and the standard normal p.d.f. 
8.1. The Expected Time to Extinction. We find the expected time to extinction $\left(E\left(\tau_{\mathrm{Q}}\right)\right)$ from quasi-stationary distribution. The expected time to extinction is given by

$$
\begin{aligned}
E\left(\tau_{\mathrm{Q}}\right) & =\frac{1}{\left(d_{I}+\delta\right) q_{.1}} \\
& =\frac{\sqrt{\sigma_{3} / N}}{\left(d_{I}+\delta\right)} \frac{\Phi\left((\widehat{I}-0.5) / \sqrt{\sigma_{3} / N}\right)}{\phi\left((1-\widehat{I}) / \sqrt{\sigma_{3} / N}\right)}
\end{aligned}
$$

We see that the expected time to extinction is a function of population size $N$, which is a function when the population size is increasing (Figure 2).

\section{Model Modification under Immune Host}

In model (4), we consider the recovery class to be joined with the susceptible host, and it may increase the population size of uninfected host. It is to be noted that sometimes for a specific disease, the recovery from infected host may not come back to the susceptible class. For such a case, after the recovery from infected host, this specific recovery class becomes immunized and can be classified into a new category. Under this assumption the modified model can be written from model (4) by just ignoring the term $\delta I$, in the growth equation of susceptible host. The algebraic manipulations for obtaining the quasi-stationary distribution and time to extinction for revised model are pretty similar with that of model (4).

\section{Numerical Illustration}

In the present study, a stochastic approach has been adopted to eradicate the infected population from a system and add on to the susceptible population. This is achieved by immunization or vaccination, and infected population recovers to susceptible population using the recovery rate $\delta$. The stochastic model in this case predicts the extinction of infection, and the time to extinction is the important measure of the persistence of infection.

Figure 1 represents the normalized simulated marginal distribution profile of the infected host $(I)$ in quasi-stationary state for $N=100, N=200$, and $N=300$. It is observed that when the population size is $N=100$, the quasi-stationary distribution is truncated and skewed, while when $N$ is increased to $N=200$ and subsequently to $N=$ 300 , the distribution displays higher kurtosis and enhanced symmetry. The population distribution of the infected class thus becomes much narrower for higher total strength of the susceptible and infected individuals. From Figure 2, we get the expected time to extinction of the infected host from quasi-stationary state when the total population $(N)$ varies from $N=100$ to $N=500$. In this case, it is observed that the expected time to extinction increases with the increase in the total population $N$. When $N$ is small, the number of infected population is also expected to be small, and at that time, the natural immune system can decelerate the rapid growth of the infected cells and add it to the susceptible class. This

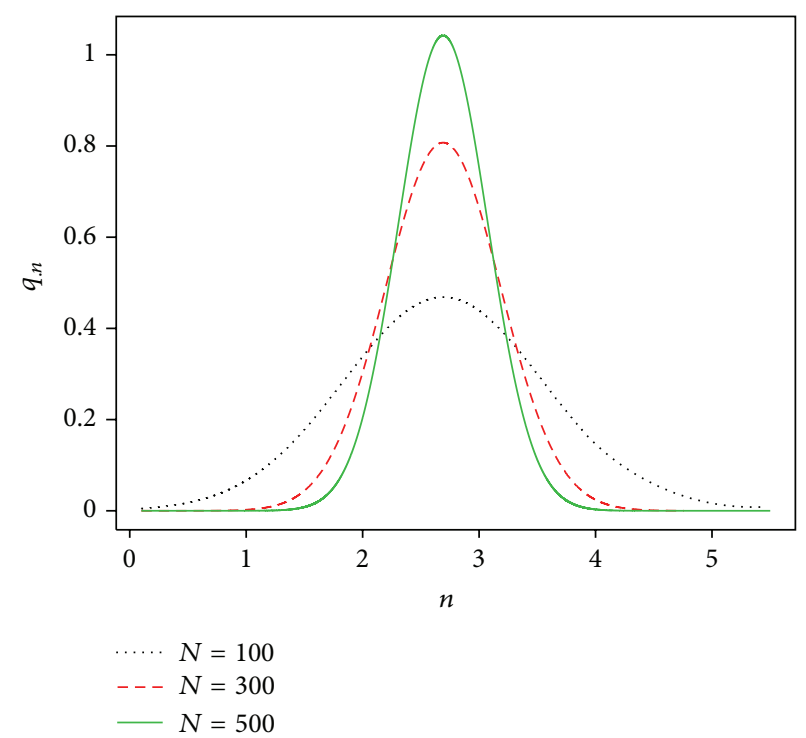

FIGURE 1: The quasi-stationary distribution for different values of $N$ and other parameters are as in Table 3.

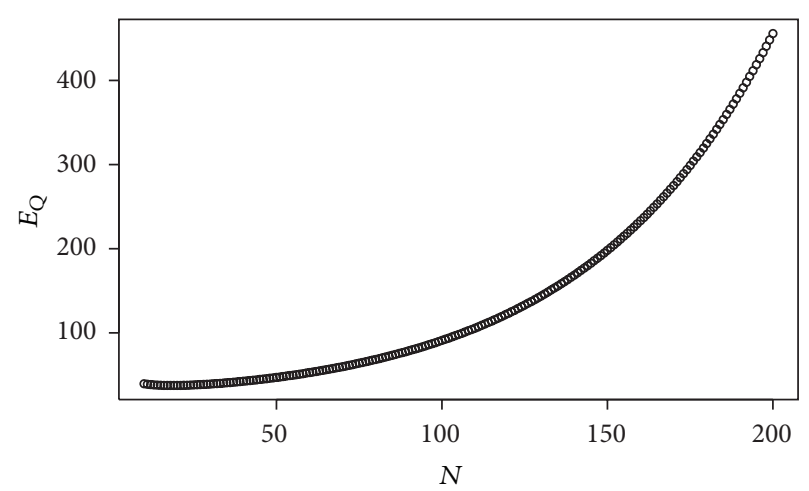

FIgURE 2: Figure depicts the expected time to extinction as a function of $N$ (10 to 200) where other parameters are as in Table 2.

results in the lesser time to extinction at the initial stages of $N$. But when the population size is high, the number of infected class is also expected to rise, and the immune system cannot control the rapid growth of the infected host population, and this leads to the high extinction time as observed. Thus, it is evident from the numerical analysis that at the onset of the time to extinction, the infected individuals get transformed and move to the susceptible population.

\section{Discussion and Conclusion}

In this paper, we have presented a basic mathematical model of host-pathogen interaction using the stochastic approach based on the concept of quasi-stationary and the diffusion approximation result. The time to extinction has been predicted as a function of the total population size $(N)$. The numerical simulation reveals that as the total population increases, the quasi-stationary distribution inclines to reduced skewness and narrower distribution of the infected 
TABLE 3: Values of parameters used in model dynamics.

\begin{tabular}{llc}
\hline Parameter Definition & $\begin{array}{c}\text { Default value } \\
\left(\text { day }^{-1}\right)\end{array}$ \\
\hline$r$ & Maximal growth rate of susceptible host & $0.2[18]$ \\
$b_{1}$ & Carrying capacity & $20[18]$ \\
$d_{1}$ & Intrinsic rate & $0.3[21]$ \\
$h_{\gamma}$ & Crowding coefficient & $0.02[21]$ \\
$\lambda$ & Half-maximal at a target cell density & $9[18]$ \\
& Force of infection through contact with & $0.2[18]$ \\
$\gamma$ & infected host & \\
$d_{I}$ & Force of infection through contact with & $0.04[18]$ \\
$\eta$ & pathogens & 2.5 liter [18] \\
$\mu$ & Pysis death rate of infected host & $115[18]$ \\
$\delta$ & Mortality rate of pathogen & $2.2[18]$ \\
\hline
\end{tabular}

class under consideration. The time to extinction of the infected class and its transformation to the susceptible class also vary with the total population size $(N)$ and are found to exhibit a gradual rise with increasing value of the total population. Since the deterministic version is an approximation of the stochastic model, the estimation of the time to extinction is unlikely to be feasible with the deterministic version. So, we are inclined to conclude that the deterministic version of the model gives an approximation to the stochastic model.

The diffusion approximation led to the conclusion that the marginal distribution of the infected host population size in quasi-stationarity is approximately $N\left(\widehat{I}, \sqrt{\sigma_{3} / N}\right)$. To achieve consistency with the fact that $I \geq 0$, the approximating normal distribution is modified by truncating at $1 / 2$. We conclude from the above result that as the total population size of the system becomes large $(N \rightarrow \infty)$, the marginal distribution of $S(t)$ is approximately $N\left(\widehat{S}, \sqrt{\sigma_{1} / N}\right)$, and the marginal distribution of $I(t)$ is approximately $N\left(\widehat{I}, \sqrt{\sigma_{3} / N}\right)$. Further, the covariance is approximated by $\sigma_{3}$. The numerical simulation confirms the analysis which is relevant in Figures 1 and 2. Further, the numerical simulation reveals that as the total population increases, the quasi-stationary distribution changes from positively skewed to symmetrical nature along with increases in the time to extinction.

The entire study has been carried out in a different angle to focus on the time to extinction of disease pathogenesis, and this reflects an advancement of our earlier reported work based on the decreasing infected population by deterministic modeling. Thus, we can conclude that stochastic modeling provides a more accurate prediction in finding out the expected time to extinction of infected population and hence disease pathogenesis.

\section{References}

[1] J. Gani, "Modelling epidemic diseases," Australian and New Zealand Journal of Statistics, vol. 52, no. 3, pp. 321-329, 2010.
[2] H. I. Freedman, "A model of predator-prey dynamics as modified by the action of a parasite," Mathematical Biosciences, vol. 99, no. 2, pp. 143-155, 1990.

[3] J. Chattopadhyay and N. Bairagi, "Pelicans at risk in Salton seaan eco-epidemiological model," Ecological Modelling, vol. 136, no. 2-3, pp. 103-112, 2001.

[4] E. Venturino, "Epidemics in predator-prey models: disease in the predators," IMA Journal of Mathematics Applied in Medicine and Biology, vol. 19, no. 3, pp. 185-205, 2002.

[5] D. Mukherjee, "Persistence in a prey-predator system with disease in the prey," Journal of Biological Systems, vol. 11, no. 1, pp. 101-112, 2003.

[6] J. Chattopadhyay, A. Mukhopadhyay, and P. K. Roy, "Effect of viral infection on the generalized Gause model of predator-prey system," Journal of Biological Systems, vol. 11, no. 1, pp. 19-26, 2003.

[7] I. Nåsell, "Stochastic models of some endemic infections," Mathematical Biosciences, vol. 179, no. 1, pp. 1-19, 2002.

[8] P. K. Roy and B. Chattopadhyay, "Host pathogen interactions with recovery rate: a mathematical study," in Proceedings of the World Congress on Engineering (WCE '10), Lecture Notes in Engineering and Computer Science, pp. 521-526, London, UK, June-July 2010.

[9] A. N. Chatterjee and P. K. Roy, "Anti-viral drug treatment along with immune activator IL-2: a control-based mathematical approach for HIV infection," International Journal of Control, vol. 85, no. 2, pp. 220-237, 2012.

[10] E. Beltrami and T. O. Carroll, "Modelling the role of viral disease in recurrent phytoplankton blooms," Journal of Mathematical Biology, vol. 32, no. 8, pp. 857-863, 1994.

[11] H. W. Hethcote and P. van den Driessche, "Two SIS epidemiologic models with delays," Journal of Mathematical Biology, vol. 40 , no. 1, pp. 3-26, 2000.

[12] R. A. Ennos, "The introduction of lodgepole pine as a major forest crop in Sweden: implications for host-pathogen evolution," Forest Ecology and Management, vol. 141, no. 1-2, pp. 8596, 2001.

[13] N. Bairagi, P. K. Roy, R. R. Sarkar, and J. Chattopadhyay, "Virus replication factor may be a controlling agent for obtaining disease-free system in a multi-species eco-epidemiological system," Journal of Biological Systems, vol. 13, no. 3, pp. 245-259, 2005.

[14] N. Bairagi, P. K. Roy, and J. Chattopadhyay, "Role of infection on the stability of a predator-prey system with several response functions-a comparative study," Journal of Theoretical Biology, vol. 248, no. 1, pp. 10-25, 2007.

[15] T. Britton and P. Neal, "The time to extinction for a stochastic SIS-household-epidemic model," Journal of Mathematical Biology, vol. 61, no. 6, pp. 763-779, 2010.

[16] I. Nåsell, "A new look at the critical community size for childhood infections," Theoretical Population Biology, vol. 67, no. 3, pp. 203-216, 2005.

[17] I. Nåsell, "On the time to extinction in recurrent epidemics," Journal of the Royal Statistical Society B, vol. 61, part 2, pp. 309330, 1999.

[18] P. K. Roy and J. Mondal, "Host pathogen interactions: insight of delay response recovery and optimal control in disease pathogenesis," Engineering Letters, vol. 18, no. 4, 2010.

[19] M. Carletti, "On the stability properties of a stochastic model for phage-bacteria interaction in open marine environment," Mathematical Biosciences, vol. 175, no. 2, pp. 117-131, 2002. 
[20] J. H. Matis, T. R. Kiffe, E. Renshaw, and J. Hassan, "A simple saddlepoint approximation for the equilibrium distribution of the stochastic logistic model of population growth," Ecological Modelling, vol. 161, no. 3, pp. 239-248, 2003.

[21] J. H. Matis, T. R. Kiffe, and P. R. Parthasarathy, "On the cumulants of population size for the stochastic power law logistic model," Theoretical Population Biology, vol. 53, no. 1, pp. 16-29, 1998. 


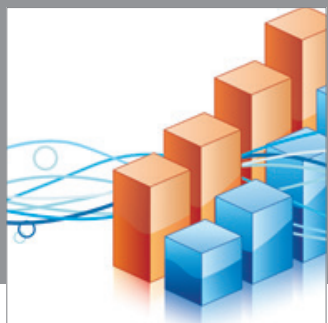

Advances in

Operations Research

mansans

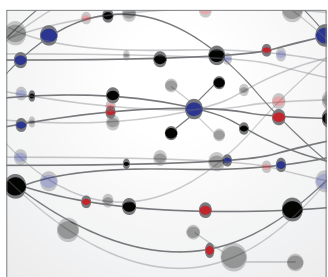

The Scientific World Journal
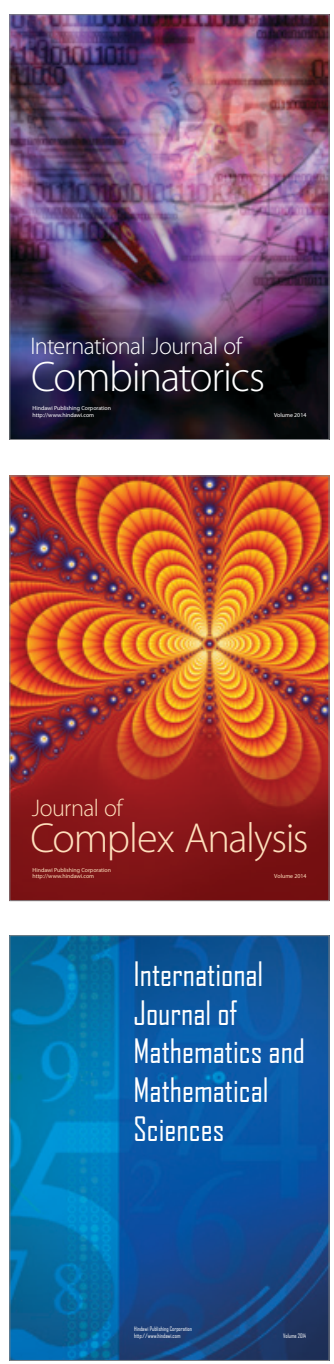
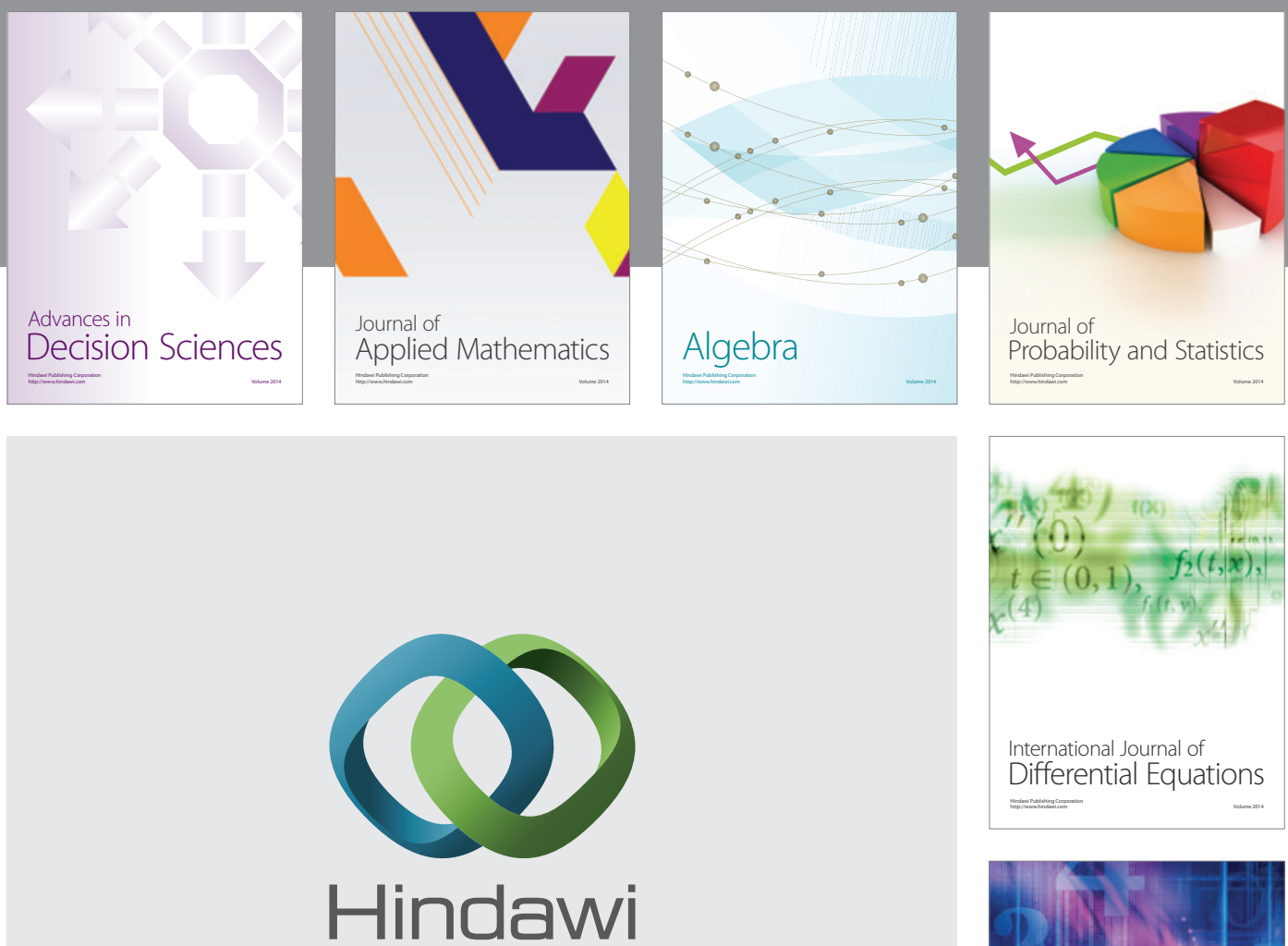

Submit your manuscripts at http://www.hindawi.com
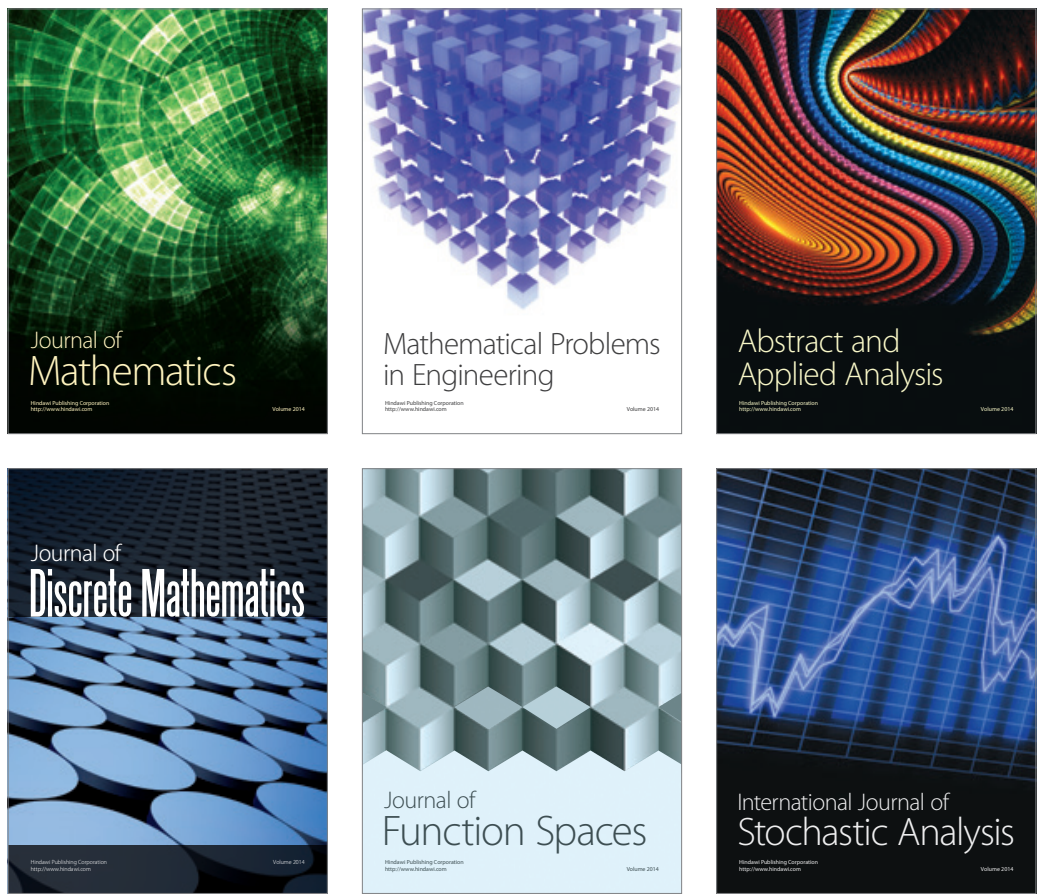

Journal of

Function Spaces

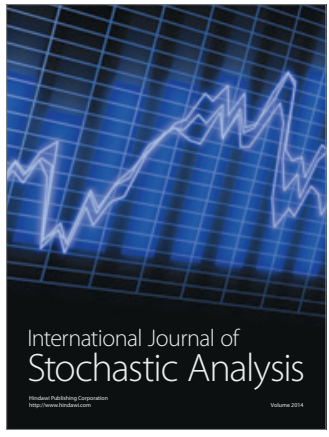

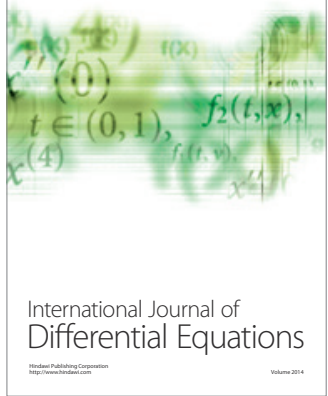
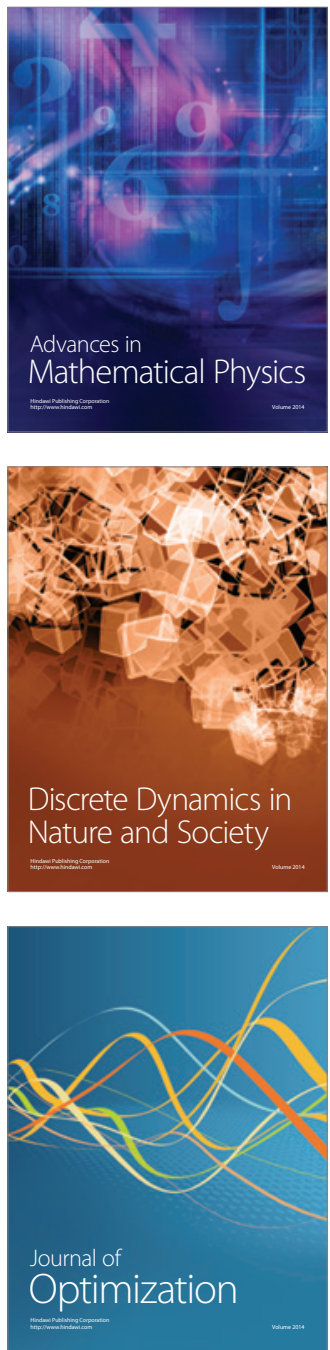